\title{
Australian Journal of \\ Crop Science \\ Virulence not linked with vegetative compatibility groups in Australian cotton Verticillium dahliae isolates
}

\section{Pearl Dadd-Daigle ${ }^{1,2}$, Karen Kirkby ${ }^{3}$, Damian Collins ${ }^{1}$, Will Cuddy ${ }^{1}$, Peter Lonergan ${ }^{3}$, Sharlene Roser ${ }^{3}$, Piklu Roy Chowdhury, ${ }^{2}$ Maurizio Labbate ${ }^{2}$ and Toni A. Chapman ${ }^{1^{*}}$}

\author{
${ }^{1}$ Biosecurity and Food Safety, New South Wales Department Primary Industries, Elizabeth Macarthur Agriculture \\ Institute, Woodbridge Road, Menangle, New South Wales, 2568, Australia \\ ${ }^{2}$ School of Life Sciences, The University of Technology Sydney, Harris Street, Ultimo, New South Wales, 2007, \\ Australia \\ ${ }^{3}$ Biosecurity and Food Safety, New South Wales Department Primary Industries, Kamilaroi Highway, Narrabri, New \\ South Wales, 2390, Australia
}

\section{Abstract}

Verticillium dahliae, the causal agent of Verticillium wilt, is a soil-borne ascomycete that infects numerous agriculturally important crops globally, including cotton. As a billion-dollar industry, cotton is economically important to Australia and the management of disease such as Verticillium wilt is key for the success of the industry. Internationally, defoliating $V$. dahliae isolates belonging to Vegetative Compatibility Group (VCG) $1 \mathrm{~A}$ cause severe damage to cotton, while non-defoliating VCG2A isolates result in significantly less disease. However, in Australia, VCG2A is causing more severe damage to crops in the field than the defoliating VCG1A. This study aimed to replicate field observations in controlled greenhouse conditions. We examined and compared disease symptoms on a range of Australian commercial cotton varieties when inoculated with different $V$. dahliae VCGs. Seedlings were root dipped in conidial suspensions and assessed over seven weeks. The final disease score, disease over time and root length were analysed. Plant mortality resulted from both $V$. dahliae VCG1A and VCG2A isolates across all cotton varieties used, confirming that there are virulent VCG2A isolates present in Australia. To our knowledge, although virulent on other plant hosts, V. dahliae VCG2A has not previously been reported to be highly virulent in cotton. We infer that virulence cannot be defined solely by VCG in Australian V. dahliae isolates causing disease in cotton.

\section{Keywords: Defoliating; Gossypium hirsutum; Non-defoliating; Verticillium; Wilt.}

\section{Introduction}

Australian farmers have been growing cotton since the 1800s (Cotton Australia, 2016). The pursuit of a plant with greater disease resistance, lower resource requirements, improved fibre quality, and higher yield has driven the development of cotton varieties. Cotton in Australia is now a billion-dollar industry, with yields increasing from approximately 9000 bales in the 1960 s to around 1.5 million bales in 2005/2006 to 2009/2010 (Cotton Australia, 2018). Australia is the third largest exporter of cotton internationally with a reputation for the highest quality of fibre produced (Cotton Australia, 2018). Disease management is now more important than ever to maintain Australia's status as an exporter of high quality cotton.

In New South Wales (NSW), the average incidence of Verticillium wilt disease ranged from 4 to $9 \%$ between $1984 / 1985$ and $1988 / 1989$ peaking at $16.6 \%$ in the $1989 / 1990$ season (Kirkby et al. 2013). Following the release of resistant varieties of cotton in 1990, disease incidence declined to $3 \%$ in 1996/1997. By 2016, the average incidence of Verticillium wilt had been gradually rising (Kirkby et al. 2016). Growers had reported disease symptoms becoming more severe with large sections of dead and dying plants, particularly in irrigated fields, resulting in large yield losses
(Jensen and Redfern 2017). CSD (2011) reported that yield losses in the Namoi Valley in NSW varied from two to four kg lint/ha for each percent of disease depending on seasonal conditions. Verticillium wilt caused losses in NSW of $\$ 1.9$ to $\$ 3.8$ million per season averaged over the five seasons up to the year 2010 (CSD 2011).

As the incidence of Verticillium wilt continues to increase, production of cotton varieties tolerant or resistant to Verticillium wilt is becoming increasingly important. In Australia, Sicala V-1 was released in 1990, followed by the release of Sicala V-2 in 1993 (Cotton Australia, 2016). Sicala $\mathrm{V}$-2 was considered a breakthrough variety in terms of yield and resistance to Verticillium wilt. However, the resistance mechanism in Sicala $V-2$ and the subsequent varieties derived from it are temperature sensitive, meaning the tolerance breaks down below $22^{\circ} \mathrm{C}$ (CSD 2011; Quinn et al. 2018). New varieties developed since the $2002 / 2003$ season are assigned a Verticillium resistance/tolerance ranking called "V-rank". The V-rank is calculated by dividing the number of test variety plants by the number of industry standard plants with symptoms in the same trial and multiplied by 100 (Salmond 2003). A higher V-rank is indicative of a more tolerant variety. Over the past decade in 
Australia, three commonly grown cotton varieties include Sicot 74BRF, 71BRF and 714B3F, which have $V$ ranks of 101, 107 , and 113 respectively.

In the United States of America, the cotton variety Acala SJ-2 was produced in 1973 to decrease the disease incidence of Verticillium wilt (Smith and Cothren 1999). Today, varieties such as FibreMax are more commonly grown. Acala SJ-2 is frequently used in international studies examining $V$. dahliae, although no longer used commercially. Acala SJ-2 does not have the resistance genes found in many modern varieties that could impact the results of virulence studies (Jiménez-Díaz et al. 2006; Korolev et al. 2001). Verticillium dahliae Kleb. is a soil-borne ascomycete that infects the vascular system of many plant species, including cotton. It is able to survive in the soil for many years in structures known as microsclerotia, which makes the management of the pathogen difficult (Davis et al. 1994). In cotton, the pathogen is classified into two pathotypes, defoliating (D) and non-defoliating (ND), and also divided into Vegetative Compatibility Groups (VCGs). The D and ND pathotypes can be identified by a simple PCR (Mercado-Blanco et al. 2003), while assigning VCG is more complex. Vegetative compatibility groups are determined by strain interaction between two nitrogen non-utilizing mutants (nit mutants) on the basis that two isolates of the same VCG have the ability to form prototrophic heterokaryons (the fusion of two genetically distinct cells) (Puhalla and Mayfield 1974). Recently, Papaioannou et al. (2013) developed a method that uses sequence data from Intergenic Spacer (IGS) regions to provide a presumptive VCG. This technique allows for faster turnaround than with the laborious production of nit mutants. For decades it was thought that only ND VCG4B was present on Australian cotton farms, but in 2014 the presence of ND VCG2A was reported by Smith et al. (2014). More recently, the presence of the D VCG1A was reported in Australian cotton for the first time (Chapman et al. 2016). At that time, VCG1A was considered exotic, however the study by Chapman et al. (2016) using isolates collected and stored in the NSW long-term culture collection revealed VCG1A had been present but undetected in Australia since at least 1983. Despite this discovery, VCG1A is not consistently causing the same extent of damage in the field as reported in other countries (Chapman et al. 2016). Additionally, severe defoliation and crop losses in Australian cotton varieties have been reported from isolates belonging to the ND VCG2A pathotype (Jensen and Redfern 2017).

There are currently no reports of ND VCG2A causing severe damage or plant mortality overseas to the same extent as seen on Australian farms, revealing a clear need to further investigate the disease presentation of Australian V. dahliae isolates. The aim of this study was to evaluate different cotton varieties for disease symptom expression when inoculated with isolates assigned as D VCG1A, ND VCG2A and ND VCG4B under controlled glasshouse conditions and to validate field observations. Cotton varieties used in this study included the American Acala SJ-2 and the Australian cotton varieties Sicot 74BRF, 71BRF and 714B3F.

\section{Results}

\section{Characterisation of Verticillium dahliae isolates}

Isolates were assigned to the groups D VCG1A, ND VCG2A, and ND VCG4B based on PCR and sequencing analysis (Table
1; Figures 1 and 2). The results indicated that isolates SS61 and SS499 were the D pathotype, while all other isolates were ND. Isolates SS61 and SS499 were assigned to VCG1A; SS362, SS285, and SS262 were assigned to VCG2A; and isolates SS364, SS289, and SS94 were assigned to VCG4B.

\section{Disease severity of infected cotton plants}

Disease severity based on a 0 - 4 scale varied between isolates of the same VCG (Table 2) (Jiménez-Díaz et al. 2016). Two D VCG1A isolates, SS61 and SS499, and one ND VCG2A isolate SS362, had significantly $(P<0.01)$ higher disease severity scores than the remaining 5 ND isolates when analysed using a generalized linear mixed model (Table 3). No defoliation was observed in any cotton variety. Plants inoculated with the D isolates SS61 and SS499 took on average 25 days to mortality, while the plants inoculated with the ND isolate SS362 took an average of 35 days (Figure 3 ; table 4). All other isolates did not cause mortality within the timeframe of the study.

\section{Disease progression over time}

The area under the disease progress stairs (AUDPS) severity scores showed significant effects caused by VCG, variety and isolate (within VCG) $(P<0.001)$. The D isolates SS499 and SS61 had the highest severity means followed by the ND isolate SS362, while Acala SJ-2 and Sicot 71BRF varieties had the highest means (Figure 4). There were no significant interactions between isolate by variety or VCG by variety ( $P$ $>0.05$ ) (Table 5).

\section{Final root length of infected cotton plants}

There were significant differences in root length of plants between variety $(P<0.05)$, but no significant differences in root length of plants infected by different VCG isolates (Figure 5). The D isolates SS61 and SS499, and the ND isolate SS362 had shorter roots than the other isolates with mean root lengths of $51.75 \mathrm{~mm}, 41.5 \mathrm{~mm}$, and $75.5 \mathrm{~mm}$, respectfully. The Acala SJ-2 and Sicot 71BRF cotton varieties had the shortest mean root lengths of $200 \mathrm{~mm}$, and 204.6 $\mathrm{mm}$, respectfully. There were no significant interactions between isolate by variety or VCG by variety $(P>0.05)$ (Table 5).

\section{Discussion}

Verticillium wilt is a persistent problem on many Australian cotton farms. Average disease incidence varies between farms with some fields presenting large patches of defoliated plants, high yield losses and plant death (Jensen and Redfern 2017). The isolates recovered from these disease-affected areas were characterised and designated as ND VCG2A, which conflicts with symptoms reported internationally (Dervis et al. 2008; Korolev et al. 2008). This study found Australian isolates from ND VCG2A vary in their ability to cause symptoms from mild foliar symptoms to plant death.

Studies that look at V. dahliae pathogenicity in cotton often use older cotton varieties such as Acala SJ-2 which are more susceptible to infection and disease (Jiménez-Díaz et al. 2016). This study found that there was a significant effect of 
Table 1. Verticillium dahliae isolates used in the glasshouse virulence assay.

\begin{tabular}{|c|c|c|c|c|c|}
\hline Herbarium number & Isolate number & Presumptive VCG & Pathotype (D/ND) & Season collected & Valley where samples were collected \\
\hline DAR82592 & SS61 & $1 A^{*}$ & $\mathrm{D}$ & $2010 / 2011$ & Namoi \\
\hline DAR83206 & SS499 & $1 \mathrm{~A}$ & D & $2015 / 2016$ & Gwydir \\
\hline DAR82597 & SS362 & $2 A^{*}$ & ND & $2013 / 2014$ & Namoi \\
\hline DAR83151 & SS285 & $2 \mathrm{~A}$ & ND & 1983/1984 & Namoi \\
\hline DAR83107 & SS262 & $2 \mathrm{~A}$ & ND & $2011 / 2012$ & Macquarie \\
\hline DAR82599 & SS364 & $4 \mathrm{~B}^{*}$ & ND & $2013 / 2014$ & Macquarie \\
\hline DAR83111 & SS289 & $4 B$ & ND & $1983 / 1984$ & Macquarie \\
\hline DAR82593 & SS94 & $4 B^{*}$ & ND & $2010 / 2011$ & Bourke/Walgett \\
\hline
\end{tabular}

*VCG determined previously using nit mutants (Chapman et al. 2016).

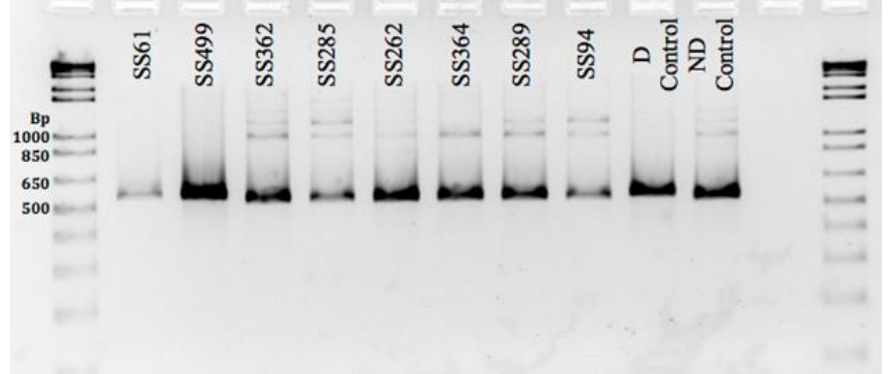

Fig 1. Visualisation of the defoliating and non-defoliating PCR as described by Mercado-Blanco, et al. (2003). Of the eight isolates, only SS61 and SS499 produced bands of $539 \mathrm{bp}$, consistent with the defoliating pathotype, while the other six isolates had bands of $523 \mathrm{bp}$, indicating the non-defoliating pathotype.

Table 2. Disease severity scores at the end of the seven week monitoring period.

\begin{tabular}{|c|c|c|c|c|c|c|}
\hline \multirow[b]{2}{*}{ Isolate } & \multicolumn{6}{|c|}{ Mean Disease Severity $(68 \% \mathrm{Cl})$} \\
\hline & Pathotype / VCG & Acala SJ-2 & Sicot 74BRF & Sicot 71BRF & Sicot 714B3F & Mean (Isolate) \\
\hline SS499 & $\mathrm{D} / 1 \mathrm{~A}$ & $4.00(-)^{\mathrm{a}}$ & $3.39(2.97-3.70)^{a}$ & $4.00(-)^{\mathrm{a}}$ & $4.00(-)^{\mathrm{a}}$ & $3.91(3.83-3.96)^{\delta \varepsilon}$ \\
\hline SS61 & $\mathrm{D} / 1 \mathrm{~A}$ & $4.00(-)^{a}$ & $3.79(3.46-3.93)^{a}$ & $3.67(3.29-3.88)^{a}$ & $4.00(-)^{a}$ & $3.92(3.82-3.97)^{\varepsilon}$ \\
\hline SS262 & $N D / 2 A$ & $1.03(0.77-1.34)^{a}$ & $0.33(0.16-0.62)^{b}$ & $0.82(0.54-1.12)^{\mathrm{ab}}$ & $0.00(-)^{\mathrm{ab}}$ & $0.52(0.36-0.71)^{\alpha}$ \\
\hline SS285 & $N D / 2 A$ & $1.98(1.58-2.37)^{b}$ & $1.53(1.21-1.91)^{\mathrm{ab}}$ & $1.57(1.24-1.97)^{\mathrm{ab}}$ & $1.07(0.79-1.38)^{\mathrm{a}}$ & $1.46(1.27-1.70)^{\beta v}$ \\
\hline SS362 & $N D / 2 A$ & $4.00(-)^{a}$ & $4.00(-)^{a}$ & $4.00(-)^{a}$ & $3.80(3.51-3.94)^{a}$ & $3.97(3.91-3.99)^{\varepsilon}$ \\
\hline SS289 & ND / 4B & $1.42(1.12-1.83)^{b}$ & $0.65(0.36-0.89)^{a}$ & $0.69(0.42-0.95)^{a}$ & $0.18(0.06-0.45)^{a}$ & $0.72(0.54-0.88)^{\alpha \beta}$ \\
\hline SS364 & $\mathrm{ND} / 4 \mathrm{~B}$ & $3.33(2.90-3.66)^{b}$ & $1.92(1.56-2.32)^{a}$ & $2.86(2.4-3.28)^{\mathrm{ab}}$ & $2.27(1.79-2.76)^{\mathrm{ab}}$ & $2.62(2.33-2.91)^{\gamma \delta}$ \\
\hline SS94 & $\mathrm{ND} / 4 \mathrm{~B}$ & $1.42(1.10-1.85)^{\mathrm{a}}$ & $1.00(0.73-1.29)^{\mathrm{a}}$ & $1.17(0.92-1.53)^{\mathrm{a}}$ & $1.00(0.74-1.30)^{\mathrm{a}}$ & $1.12(0.97-1.33)^{\alpha \beta}$ \\
\hline $\begin{array}{l}\text { Mean } \\
\text { (Variety) }\end{array}$ & & $3.14(2.92-3.33)^{\gamma}$ & $2.03(1.84-2.24)^{\alpha \beta}$ & $2.54(2.32-2.76)^{\beta}$ & $1.88(1.71-2.07)^{\alpha}$ & \\
\hline
\end{tabular}

Letters within the table compare varieties within each isolate. Greek letters for the $\mathrm{m}$
scores on the boundary $(0$ or 4$)$, a reliable $\mathrm{Cl}$ cannot be computed, and so is not shown.

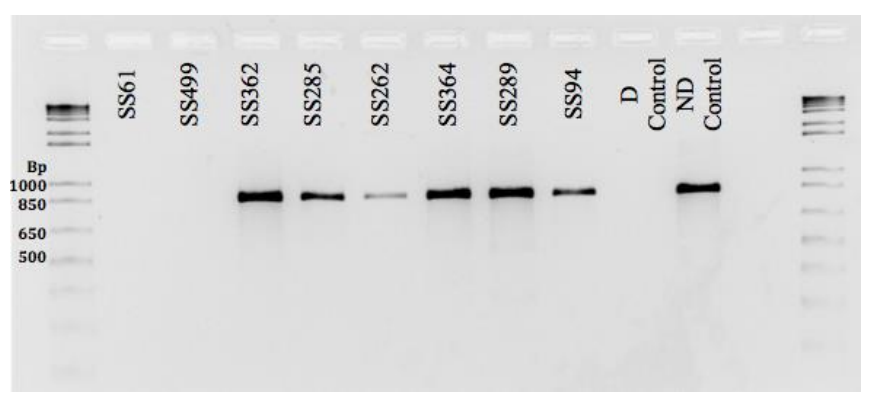

Fig 2. Visualisation of the confirmatory defoliating and non-defoliating PCR as described by Mercado-Blanco et al. (2001). Isolates SS362, SS285, S262, SS364, SS289, and SS94 all produced bands of 824 bp in size, indicating that they are of the non-defoliating pathotype. Amplification of isolates SS61 and SS499 both resulted in no product being produced, consistent with the defoliating pathotype. 
Table 3. Wald tests for fixed terms in the ordinal GLMM for disease severity.

\begin{tabular}{lllll}
\hline & DF & Den. DF & F-stat. & P-value \\
\hline VCG & 2 & 8.55 & 9.1696 & 0.0075 \\
Variety & 3 & 705 & 6.2602 & $<0.001$ \\
VCG/Isolate & 5 & 8.99 & 8.0966 & 0.0038 \\
VCGxVariety & 6 & 705 & 0.261 & 0.9548 \\
VCG/IsolatexVariety & 15 & 705 & 0.4196 & 0.9738 \\
\hline
\end{tabular}

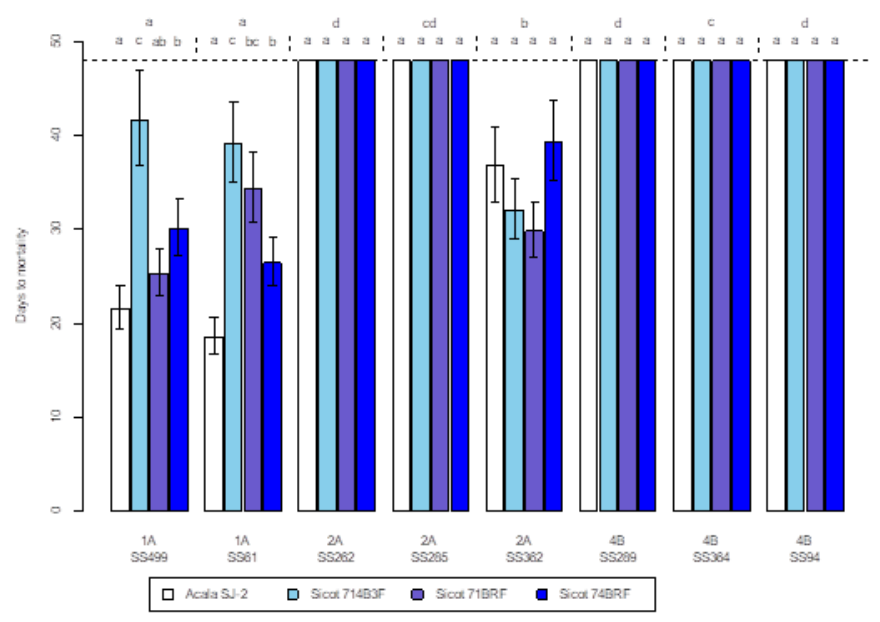

Fig 3. Days to mortality. The Number of days until cotton plant varieties died following infection with mortality-causing $V$. dahliae isolates. Letters indicate significant differences between isolates and differences between varieties within isolate. Error bars show standard error.

Table 4. Analysis of deviance table for the parametric survival regression for days to mortality.

\begin{tabular}{llllll}
\hline & Df & Deviance & Resid. Df. & $-2 \times$ LL & P-value \\
\hline VCG & & & 183 & 751.22 & \\
Variety & 2 & 130.90 & 180 & 620.32 & $<0.001$ \\
VCG/Isolate & 3 & 11.61 & 177 & 608.71 & 0.009 \\
VCG $\times$ Variety & 5 & 85.56 & 149 & 523.15 & $<0.001$ \\
\hline
\end{tabular}
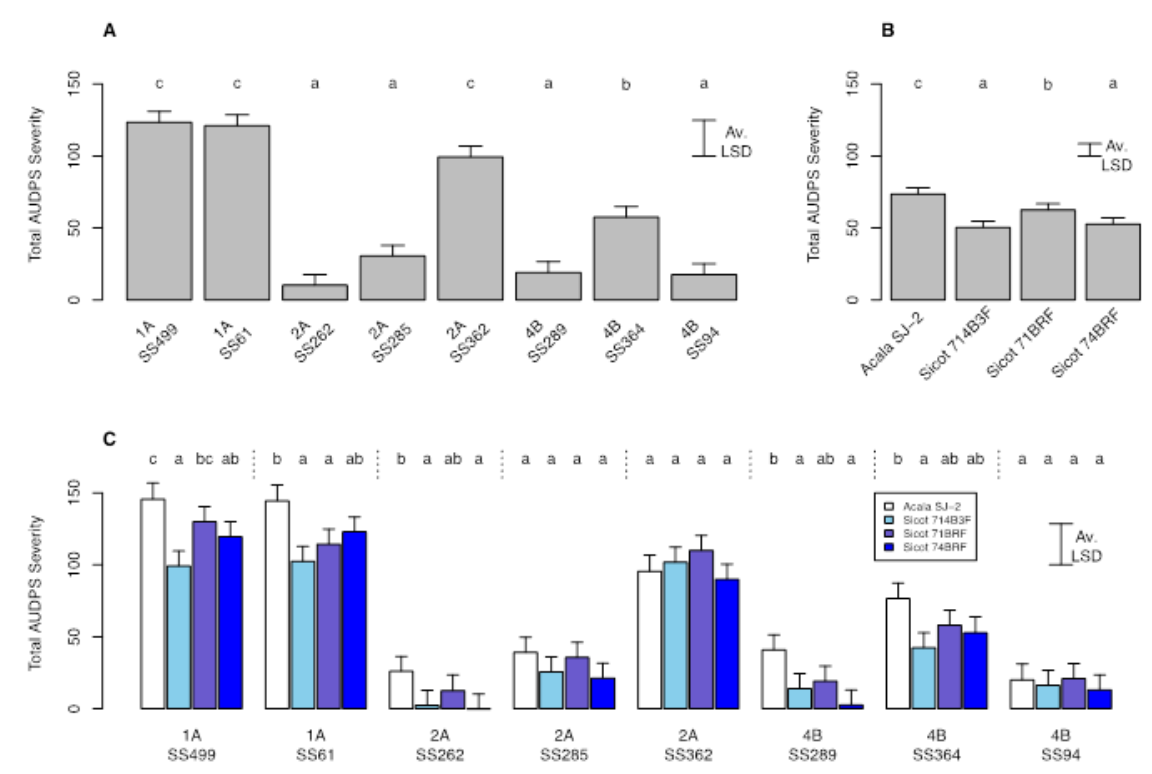

Fig 4. Total AUDPS severity. (A) AUDPS means for each isolate. (B) AUDPS means for each cotton variety. (C) AUDPS means for each isolate by variety. Av LSD indicates average least significant difference. Different lower case letters indicate significant difference between bars. 
Table 5. Wald F-test statistics for fixed terms in the model.

\begin{tabular}{lllll}
\hline AUDPS & DF & DenDF & F-stat & P-val \\
\hline VCG & 2 & 5.8 & 100.4000 & $<0.001$ \\
Variety & 3 & 112.5 & 12.0400 & $<0.001$ \\
VCG $\times$ Isolate & 5 & 6.3 & 20.8000 & $<0.001$ \\
VCG $\times$ Variety & 6 & 112.6 & 1.9430 & 0.080 \\
VCG $\times$ Isolate $\times$ Variety & 15 & 112.6 & 0.7364 & 0.743 \\
Log10 Root length & & & & \\
\hline & DF & DenDF & F-stat & P-val \\
VCG & 3 & 3.1 & 5.100 & 0.105 \\
Variety & 3 & 77.3 & 5.955 & 0.001 \\
VCG $\times$ Isolate & 5 & 4.2 & 5.993 & 0.050 \\
VCG $\times$ Variety & 9 & 76.9 & 1.423 & 0.193 \\
VCG $\times$ Isolate $\times$ Variety & 15 & 77.9 & 1.445 & 0.148 \\
\hline
\end{tabular}
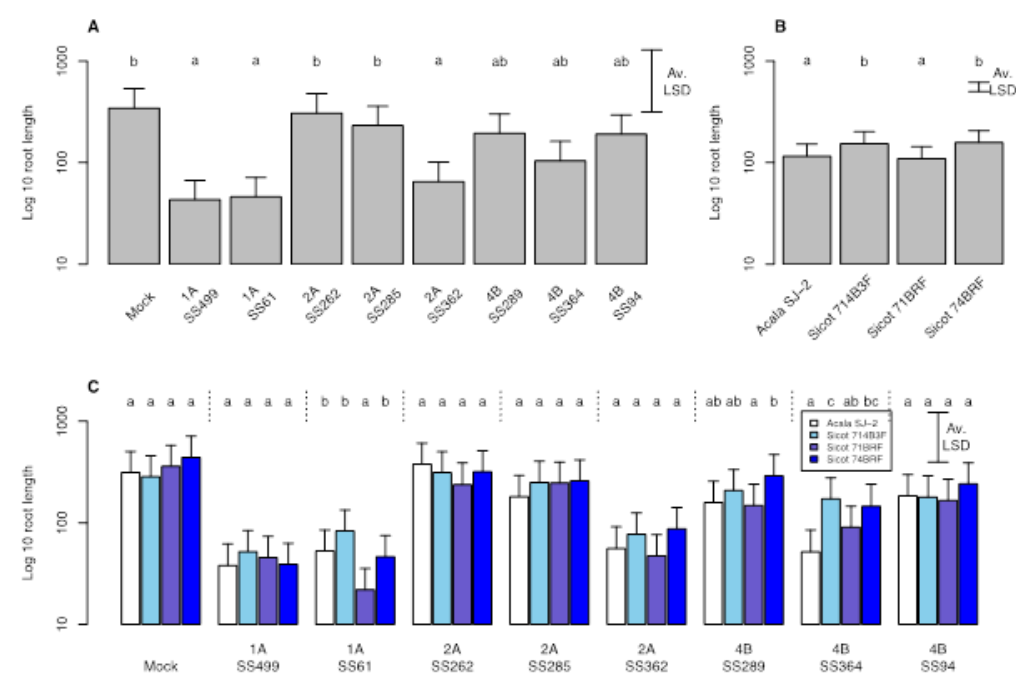

Fig 5. The average root length of infected plants. A) Average root lengths for each isolate. B) Average root lengths for each cotton variety. C) Average root lengths for each isolate by variety. Av LSD indicates average least significant difference. Different lower case letters indicate significant difference between bars.

Table 6. Cotton varieties used in the glasshouse virulence assay.

\begin{tabular}{lll}
\hline Cotton Variety & Description & V-rank \\
\hline Acala SJ-2* & Developed in 1973 to help improve crop resistance to & \\
& Verticillium wilt & 107 \\
Sicot 71BRF** & Bollgard II ${ }^{\circledR}$ stacked with Roundup Ready Flex ${ }^{\circledR}$ & 101 \\
Sicot 74BRF** & Bollgard II ${ }^{\circledR}$ stacked with Roundup Ready Flex & 113 \\
\hline Sicot 714B3F** & Bollgard ${ }^{\circledR}$ stacked with Roundup Ready Flex ${ }^{\circledR}$ & \\
\hline${ }^{*}$ No V-rank established for Acala SJ-2; ${ }^{* *}$ Australian varieties of cotton.
\end{tabular}

the disease progress over time (AUDPS scores) for the varieties, as Acala SJ-2 was more susceptible across the board. The interaction between isolate and variety was not significant as disease outcomes were fairly consistent across plants infected with the same isolate regardless of cotton variety. However, the effects of both VCG and isolate within VCG were significant $(P<0.01)$. This indicated that while there is a difference in disease progress over time between the VCGs, there is variation between isolates within each of the VCGs. For this reason, in Australia, knowing the VCG of a $\mathrm{V}$. dahliae isolate is not an effective measure of V. dahliae pathogenicity or useful for predicting potential disease outcomes. This is evident by the fact that plants infected with the ND VCG2A isolate SS362 caused plant mortality at the same level of severity as plants infected with the $D$
VCG1A isolates SS499 and SS61. The root lengths were equally consistent with the AUDPS and disease severity scores. Plants that had higher AUDPS and higher disease severity scores had shorter roots, indicating that they had either spent fewer resources towards growing or died before the roots could get larger. The two more susceptible cotton varieties also had shorter roots lengths, consistent with expectations for susceptible varieties. The lack of a significant interaction between isolates and cotton variety also points to the isolate being a key factor in the determination of plant disease outcome. Additionally, these results show that while the recent cotton varieties used in Australia can slow disease progression, they do not make a notable impact on disease outcome regardless of the VCG type. 
Observations made in fields, where the ND VCG2A isolates have been obtained from, varied from no defoliation, mild to severe foliar symptoms, to complete defoliation as well as plant death despite being a ND pathotype (Jensen and Redfern 2017). However, plants in this study did not defoliate when infected with any of the isolates, including the D VCG1A SS61 which has previously been shown to defoliate (Jiménez-Díaz et al. 2016). This defoliation did not appear significant given mortality was observed in both previous studies. In the field VCG1A causes minimal disease symptoms (Chapman et al. 2016). In our study, both D SS61 and D SS499 were highly virulent and resulted in plant death in the least amount of time compared to the other VCGs. This could be contributed to the infection method as root dipping introduces conidia into the plant at concentration levels that may be higher than natural infection. Natural infection also relies on conidia and hyphae germinating and infecting a plant root. Infection percentage is guaranteed when artificially inoculating plants and the number of conidia inside the plant are also higher.

Greenhouse trials are designed with the intent to produce pathogenicity results providing the optimum conditions for the pathogen and not necessarily the host plant. The root dipping method, with conidia as the only inoculum source, used in this study (Trapero et al. 2013) is not representative of the natural infection process. The consistent temperature of the greenhouse also differs from the hot days and cold nights that plants are subjected to on cotton farms. The difference in these conditions could account for some of the disparity.

Previous research suggests that the separate VCGs of V. dahliae are generally virulent on different hosts. While VCG1A is known to be virulent in cotton and olives (Dervis et al. 2007), VCG2A is generally virulent in tomatoes (Tsror et al. 2001), and VCG4B is virulent in mint and potato (Dung et al. 2013). In Israel however, the ND VCG2B is the most pathogenic $\mathrm{V}$. dahliae in cotton rather than VCG1A (Korolev et al. 2001). Verticillium dahliae is an adaptable pathogen, and has been shown to have a high number of translocatable elements, allowing it to rearrange its genetic material in response to new hosts (de Jonge et al. 2013). It is likely that a subset of Australian VCG2A V. dahliae isolates may have adapted from a separate host, have acquired additional virulence genes from another source (Chen et al. 2017), or have simply mutated as a response to the continuous selective pressure of growing cotton. No obvious morphological differences between virulent and nonvirulent isolates have been found (data not shown). As virulence is currently determined through in vivo assays and field reports, genome analysis and better molecular tools for the analysis of virulence would be useful to further understand the Australian VCG2A.

\section{Materials and Methods}

\section{Verticillium dahliae isolates and molecular characterisation}

Eight V. dahliae isolates derived from single microsclerotia initially collected from diseased cotton plants in fields with varying disease symptoms throughout NSW were selected from the Australian Cotton Research Institute Pathology Long Term Culture Collection stored in Narrabri. These isolates were also lodged with the NSW DPI Plant Pathology and Mycology Herbarium in Orange and given unique DAR identification numbers (Table 1). The isolates were grown on Potato Dextrose Agar (PDA) for two weeks at $23^{\circ} \mathrm{C}$ before DNA was extracted using a QIAGEN DNeasy extraction kit (cat no 69106; Venlo, The Netherlands).

Isolates were characterised as either D or ND pathotypes. Pathotypes were identified by 539 bp (D) or 523 bp (ND) bands observed on an electrophoresis gel (Figure 1). The PCR was run as described by Mercado-Blanco, et al. (2003). Non-defoliating strains were further distinguished by $824 \mathrm{bp}$ band (Figure 2) using the method described by MercadoBlanco, et al. (2001). Four isolates with VCGs determined in a previous study (Chapman et al. 2016) were included as reference isolates. Presumptive VCGs were determined for the remaining isolates using the variable IGS region which were amplified via PCR to give a $1600 \mathrm{bp}$ segment of DNA, as described by Qin, et al. (2006). The resulting product was cleaned using a QIAquick PCR purification kit (Qiagen), DNA concentration confirmed using the NanoDrop, and sent for sequencing at the Westmead Millennium Institute, NSW Australia. The sequence was then analysed using Geneious version 9.1.5 (https://www.geneious.com, Kearse et al. 2012).

\section{Pathogenicity assay}

Each of the isolates were grown in Potato Dextrose Broth at room temperature constantly shaking at 180 RPM for seven days. Conidial suspensions were subsequently filtered through four layers of sterile cheesecloth and adjusted to a concentration of $1 \times 106$ conidia/ml following microscopic counts using a haemocytometer (Trapero et al. 2013). Cotton seeds encompassing four varieties (Table 6) were triple washed with sterile milli-Q water, and germinated on blotting paper for 72 hours. Single germinated seeds were planted in root trainers $12 \mathrm{~cm} \times 4 \mathrm{~cm}$ containing twice pasteurised Debco native potting mix supplemented with Osmocote (N:P:K ratio of 21.2:1.9:5.7; manufactured by Scotts Australia PTY LTD, NSW, Australia) at the recommended rate of $1 / 2$ a tablespoon per perennial plant. Seedlings at the two true leaf stage were gently removed from the root trainers and loose soil removed from the roots, and inoculated via root dipping in conidial suspension. One centimeter of the root was cut from the base of each tap and lateral root before the seedlings were immersed in the conidial suspensions or sterile water for 25 minutes. Seedlings were then transplanted into $175 \mathrm{~mm}$ pots containing twice pasteurised Debco native potting mix supplemented with the recommended rate of Osmocote. The seedlings were arranged in a randomised complete block design with the six replicates, each forming one block, spread out over two rooms, and observed for seven weeks. Plants were maintained in a glasshouse at a temperature of $22 \pm 2^{\circ} \mathrm{C}$, and watered as needed.

\section{Disease assessment}

The disease severity of each plant was recorded bi-weekly over seven weeks using a $0-4$ scale monitoring the external foliar symptoms for a total of 14 assessments (Jiménez-Díaz et al. 2016). A score of 0 indicated no external foliar symptoms, "1" was 1 - 33\% of leaves with foliar symptoms, "2" indicated 34 - 66\% of leaves affected, "3" was $67-99 \%$ 
affected, and " 4 " indicated $100 \%$ disease and plant death. Disease severity over time was quantified for each individual plant over the seven weeks post inoculation using the area under the disease progress stairs (AUDPS). The AUDPS was calculated using the trapezoidal method (Simko and Piepho 2011) using the formula:

"AUDPS=" ["y" _"1" "x" \{("t" _"2 " "-" "t" _"1" )/"2" "+" "D"

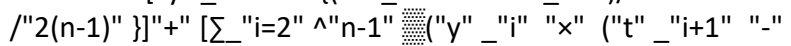
"t" _"i-1" )/"2" ) ]"+" ["y" _"n" "x" \{("t" _ "n" "--" "t" _"n-1" )/"2" "+" "D" /"2(n-1)" \}]" "

where $\{y 1\}$ and $\{y n\}=$ assessments at the first and last observations

$\{\mathrm{t} 1\},\{\mathrm{t} 2\},\{\mathrm{tn}-1\}$, and $\{\mathrm{tn}\}=$ times of the first, second, penultimate and final observations, respectively

$\mathrm{D}=\mathrm{tn}-\mathrm{t} 1$

$\mathrm{n}=$ the total number of observations

\section{Root measurements}

Roots from each plant were cut at ground level and soil removed by rinsing under running water. The clean roots were placed in paper envelopes and stored until analysis was undertaken. Each root sample was individually imaged by placing the roots of a single plant flat in a clear tray, the roots separated, and distilled water added until all the roots were just covered. The roots were scanned and the resulting image was analysed to estimate root length using WinRHIZO Pro V. 2009c (Regent Instruments Canada INC) (Arsenault et al. 1995). This was repeated for each collected root sample.

\section{Statistical analysis}

Linear mixed models were fitted to the AUDPS and log10 Root length and a generalized linear mixed model (GLMM) for disease severity with an ordinal response. For days to mortality, a parametric survival regression was fitted. For AUDPS, disease severity and days to mortality, data for the "mock" strain was excluded from analysis as it was all zero. Each model consisted of the fixed effects of VCG, isolate (within VCG), variety and all interactions, and, for the mixed models, random effects of room, replicate and their interactions with VCG, isolate and variety. All mixed models were fitted using ASReml-R (Butler 2018) in the R statistical environment, version 3.6 ( $R$ Core Team 2019). The parametric survival regression for days to mortality was fitted using the survreg function in the survival package (Therneau 2015). Pairwise comparisons for disease severity and days to mortality were determined using Wald and likelihood ratio tests respectively (using a dummy variable to create an appropriate reduced model). For disease severity, a mean score was computed (Hannah and Quigley 1996) with a $68 \% \mathrm{Cl}$ (corresponding to $\pm 1 \mathrm{SE}$ for a normal distribution) calculated using Monte Carlo simulation with 1000 simulations.

\section{Conclusion}

On Australian cotton farms, both D VCG1A and ND VCG2A isolates are capable of causing disease and significant yield loss. It is not clear why VCG1A is not exhibiting the extensive disease symptoms on Australian farms as reported overseas. The ND VCG2A isolates on the other hand are causing a spectrum of disease symptoms ranging from mild foliar symptoms to plant death both in the field and in this study. However, ND VCG4B isolates cause less severe disease symptoms. It appears that, at least in Australia, VCGs are not a useful pathogenicity indicator of $\mathrm{V}$. dahliae in cotton and an alternate classification system is needed.

\section{Acknowledgements}

This project is supported by funding from the Australian Government Department of Agriculture and Water Resources as part of its Rural R\&D for Profit programme and the Cotton Research and Development Corporation. All cotton seed was supplied by Cotton Seed Distributors. Bernie Dominiak reviewed and improved an earlier version of this manuscript.

\section{References}

Arsenault JL, Poulcur S, Messier C, Guay R (1995) WinRHIZO'm, a root-measuring system with a unique overlap correction method. HortSci. 30:906D-906 doi:10.21273/HORTSCI.30.4.906D

Butler D (2018) asreml: Fits the Linear Mixed Model. URL www.vsni.co.uk. R package version. 4.1.0.98.

Chapman TA, Chambers GA, Kirkby K, Jiménez-Díaz RM (2016) First report of the presence of Verticillium dahliae VCG1A in Australia. Australasian Plant Disease Notes. 11:14

Chen JY, Liu C, Gui YJ, Si KW, Zhang DD, Wang J, Short DPG, Huang JQ, Li NY, Liang Y, Zhang WQ, Yang L, Ma XF, Li TG, Zhou L, Wang, BL, Bao YM, Subbarao KV, Zhang GY, Dai XF (2017) Comparative genomics reveals cotton-specific virulence factors in flexible genomic regions in Verticillium dahliae and evidence of horizontal gene transfer from Fusarium. New Phytologist. 217:756-770

Cotton Australia (2016) Cotton Annual 2016. Australian Cotton Industry Statistics.

Cotton Australia (2018) Cotton Annual 2018. Australian Cotton Industry Statistics.

CSD (2011) Cotton Seed Distributors. Seeds for Thought. Verticillium wilt update, vol 10. Wee Waa, Australia

Davis JR, Pavek JJ, Corsini DL, Sorensen LH, Schneider AT, Everson DO, Westermann DT, Huisman OC (1994) Influence of continuous cropping of several potato clones on the epidemiology of Verticillium wilt of potato Phytopathology. 84:207-214.

De Jonge $R$, Bolton MD, Kombrink A, van den Berg GCM, Yadeta KA, Thomma BPHJ (2013) Extensive chromosomal reshuffling drives evolution of virulence in an asexual pathogen. Genome Research. 23:1271-1282.

Dervis S, Erten L, Soylu S, Tok FM, Kurt S, Yıldız M, Soylu EM (2007) Vegetative compatibility groups in Verticillium dahliae isolates from olive in western Turkey. European Journal of Plant Pathology. 119:437-447.

Dervis S, Kurt S, Soylu S, Erten L, Mine Soylu E, Yıldız M, Tok FM (2008) Vegetative compatibility groups of Verticillium dahliae from cotton in the southeastern anatolia region of Turkey. Phytoparasitica. 36:74-83.

Dung JKS, Peever TL, Johnson DA (2013) Verticillium dahliae Populations from mint and potato are genetically divergent with predominant haplotypes. Phytopathology. 103:445-459. 
Hannah M, Quigley P (1996) Presentation of ordinal regression analysis on the original scale biometrics. 52:771-775.

Jensen M, Redfern R (2017) Breaking the Verticillium cycle vol Winter 2017. Cotton Research and Development Corporation (CRDC).

Jiménez-Díaz RM, Mercado-Blanco J, Olivares-García C, Collado-Romero M, Bejarano-Alcázar J, Rodríguez-Jurado D, Giménez-Jaime A, García-Jiménez J, Armengol J (2006) Genetic and Virulence Diversity in Verticillium dahliae Populations Infecting Artichoke in Eastern-Central Spain. Phytopathology. 96:288-298

Jiménez-Díaz RM, Olivares-García C, Trapero-Casas JL, Jiménez-Gasco MM, Navas-Cortés JA, Landa BB, Milgroom MG (2016) Variation of pathotypes and races and their correlations with clonal lineages in Verticillium dahliae. Plant Pathology. 66:651-666

Kearse $M$, Moir R, Wilson A, Stones-Havas $S$, Cheung $M$, Sturrock S, Buxton S, Cooper A, Markowitz S, Duran C, Thierer T, Ashton B, Meintjes P, Drummond A (2012) Geneious Basic: an integrated and extendable desktop software platform for the organization and analysis of sequence data Bioinformatics (Oxford, England) 28:16471649.

Kirkby K, Lonergan P, Cooper B, Roser S (2016) Diseases of Cotton XI Final Report. Narrabri, Australia.

Korolev N, Pérez-Artés E, Bejarano-Alcázar J, RodríguezJurado D, Katan J, Katan T, Jiménez-Díaz RM (2001) Comparative study of genetic diversity and pathogenicity among populations of Verticillium dahliae from cotton in Spain and Israel. European Journal of Plant Pathology. 107:443-456.

Mercado-Blanco J, Rodríguez-Jurado D, Parrilla-Araujo S, Jiménez-Díaz RM (2003) Simultaneous detection of the defoliating and non-defoliating Verticillium dahliae pathotypes in infected olive plants by duplex, nested polymerase chain reaction. Plant Disease. 87:1487-1494.

Mercado-Blanco J, Rodríguez-Jurado D, Pérez-Artés E, Jiménez-Díaz RM (2001) Detection of the non-defoliating pathotype of Verticillium dahliae in infected olive plants by nested PCR. Plant Pathology. 50:609-619.
Papaioannou IA, Dimopoulou CD, Typas MA (2013) Structural and phylogenetic analysis of the rDNA intergenic spacer region of Verticillium dahliae. FEMS Microbiology Letters. 347:23-32.

Puhalla JE, Mayfield JE (1974) The Mechanism of heterokaryotic growth in Verticillium dahliae. Genetics. 76:411-422.

Qin Q-M, Vallad GE, Wu BM, Subbarao KV (2006) Phylogenetic analyses of phytopathogenic isolates of Verticillium spp. Phytopathology. 96:582-592.

Quinn J, Eveleigh R, Ford B, Millyard J, Teague C, Barry C, Lee S, Devlin A, McDonald C (2018) Verticillium Wilt. Facts on Friday vol October. Cotton Seed Distributors, Wee Waa, Australia.

R Core Team (2019) R: A Language and Environment for Statistical Computing. R Foundation for Statistical Computing, Vienna, Austria, 2019. URL http://www.Rproject.org

Salmond G (2003) Disease ratings: another management tool for cotton growers Cotton Grower. 24:9

Simko I, H-P Piepho (2011) The area under the disease progress stairs: calculation, advantage, and application. Phytopathology. 102: 381-389.

Smith CW, Cothren JT (1999) Cotton: origin, history, technology, and production. Wiley Series in Crop Science. John Wiley \& Sons.

Smith L, Scheikowski L, Bauer B, Lahane J, Allen S (2014) Pathogens in Australian cotton. paper presented at the 17th Australian Cotton Conference, Gold Coast, Australia 2014.

Therneau TM (2015). A Package for Survival Analysis in S. URL https://CRAN.R-project.org/package=survival. version 2.38 .

Trapero C, Díez CM, Rallo L, Barranco D, López-Escudero FJ (2013) Effective inoculation methods to screen for resistance to Verticillium wilt in olive Scientia. Horticulturae. 162:252-259.

Tsror L, Hazanovsky M, Mordechi-Lebiush S, Sivan S (2001) Aggressiveness of Verticillium dahliae isolates from different vegetative compatibility groups to potato and tomato. Plant Pathology. 50:477-482. 